\title{
Inzidenz und Prognose
}

Fragestellung: Wie ist die Prognose von Patienten mit kryptogener TIA oder kryptogenem ischämischen Insult?

Hintergrund: $20 \%$ aller TIA und ischämischen Infarkte sind ungeklärter Ätiologie und werden als kryptogen eingestuft. Der Prozentsatz kryptogener Schlaganfälle ist bei unter 65-Jährigen höher als bei älteren Menschen. Es gibt relativ wenig populationsbezogene Studien zur Inzidenz und Prognose.

Patienten und Methodik: Die Oxford Vascular Study analysierte die Daten von 92.728 Personen, die bei 100 praktischen Ärzten in England registriert sind. Die Studie lief von 2002 bis 2014. Verglichen wurden Patienten mit kryptogener TIA und ischämischen Insult mit Patienten mit anderen Schlaganfallätiologien.

Ergebnisse: Von den 2.555 Patienten mit TIA und Schlaganfall hatten 812 (32\%), ein kryptogenes Ereignis. Tod oder Pflegebedürftigkeit nach sechs Monaten betrugen $23 \%$ bei den kryptogenen Schlaganfällen und $27 \%$ bei Makro- und Mikroangiopathie. Patienten mit kryptogenem Schlaganfall hatten weniger arteriosklerotische Risikofaktoren (arterielle Hypertonie, Diabetes mellitus, periphere arterielle Verschlusskrankheit, Hypercholesterinämie und Rauchen) als Patienten mit Makroangiopathie, Mikroangiopathie und kardioembolischen Schlaganfällen.

Li L, Yiin GS, Geraghty OC, Schulz UG et al; Oxford Vascular Study. Incidence, outcome, risk factors, and long-term prognosis of cryptogenic transient ischaemic attack and ischaemic stroke: a population-based study. Lancet Neurol 2015; 14 : $903-13$

\section{Hier steht eine Anzeige.}

Schlussfolgerungen: Etwa ein Drittel aller Schlaganfälle ist kryptogen. Die Prognose ist ähnlich schlecht wie bei Makro- und Mikroangiopathie, offenbar spielt aber eine Arteriosklerose in der Pathophysiologie eine deutlich geringere Rolle als bei anderen Schlaganfallmechanismen.

- Kommentar von Hans-Christoph Diener, Essen

\section{Spielen Embolien eine Rolle?}

Dies ist die größte bisher publizierte populationsbezogene Studie zur Prognose kryptogener TIA und Schlaganfälle. Dass diese Patienten deutlich weniger Risikofaktoren für eine Arteriosklerose haben, könnte dafür sprechen, dass embolische Mechanismen hier eine Rolle spielen. In der Studie selbst wurde allerdings klinisch stummes paroxysmales Vorhofflimmern relativ selten entdeckt. Die Daten würden aber dafür sprechen, dass es eine Rationale dafür gibt, bei Patienten mit embolischem Schlaganfall ungeklärter Ätiologie (ESUS) eine orale Antikoagulation mit neuen Antikoagulanzien mit Acetylsalicylsäure zu vergleichen. Zwei dieser Studien (RESPECT-ESUS mit Dabigatran und NAVIGATE-ESUS mit Rivaroxaban) untersuchen zurzeit dieses Konzept. 